\title{
Pain Behavior Changes Following Disc Puncture Relate to Nucleus Pulposus Rather than to the Disc Injury Per Se: An Experimental Study in Rats
}

\author{
Elin Nilsson ${ }^{1}$, Toshio Nakamae ${ }^{2,3}$ and Kjell Olmarker ${ }^{*}, 3$ \\ ${ }^{I}$ Department of Orthopaedics, Inst Clinical Sciences, Sahlgrenska Academy, University of Gothenburg, Gothenburg, \\ Sweden \\ ${ }^{2}$ Department of Orthopaedic Surgery, Graduate School of Biomedical Sciences, Hiroshima University, Japan \\ ${ }^{3}$ Musculoskeletal research, Department of Medical Chemistry and Cell Biology, Institute of Biomedicine, Sahlgrenska \\ Academy, University of Gothenburg, Gothenburg, Sweden
}

\begin{abstract}
It has previously been demonstrated that disc puncture in the rat induced changes in grooming and wet dog shakes, two behavioral changes that may be linked to discomfort and neuropathic pain. In this study the aim was to separate the effects of disc injury and the epidural presence of nucleus pulposus. Following anesthesia, the L4-5 disc was exposed using a dorsal approach. Ten rats received a superficial disc injury without nucleus pulposus leakage and ten rats received nucleus pulposus from a donor rat without disc injury. In ten animals the L4-5 disc was punctured using a ventral approach, with 10 corresponding controls. Spontaneous behavior was assessed after surgery. The data was matched to historical control of dorsal sham surgery and disc puncture. The study showed that the effects of nucleus pulposus were more pronounced than the effects induced by the disc injury. Ventral disc puncture did not induce any behavioral changes different from sham exposure. In conclusion, the data from the study indicate that behavioral changes induced by disc puncture are more likely to relate to the epidural presence of nucleus pulposus than the disc injury per se.
\end{abstract}

Keywords: Low back pain, disc, nucleus pulposus, rat, pain behavior.

\section{INTRODUCTION}

It was recently experimentally demonstrated that an isolated puncture of a lumbar disc, without simultaneous mechanical deformation, may induce certain pain behavior changes in the rat [1]. In particular, the rats demonstrated an increased grooming and "wet dog shakes", two behaviors that have been linked to the presence of neuropathic pain [28]. From the data, however, it was not understood if the changes were induced by the disc injury or by the intra spinal presence of nucleus pulposus. In an attempt to isolate the effects of disc injury versus exposure to nucleus pulposus, the same behavioral changes were studied following disc injury without nucleus pulposus leakage, application of nucleus pulposus without disc injury, and both disc injury and nucleus pulposus exposure through a ventral disc puncture. The data was matched to historical control of sham surgery and dorsal disc puncture.

\section{MATERIALS AND METHODOLOGY}

Forty female Sprague-Dawley rats (Charles River Laboratories, Solna, Sweden) with an average bodyweight of $225 \mathrm{~g}$ were housed in groups of four with free access to food (B\&K Rat/mouse standard, BeeKay feeds \& beddings, Sollentuna, Stockholm) and tap water. Temperature was kept

*Address correspondence to this author at the Department of Medical Chemistry and Cell Biology, Institute of Biomedicine, Sahlgrenska Academy, University of Gothenburg, SE - 40530 Gothenburg, Sweden; Tel: +46-31-7863597; E-mail: kjell.olmarker@gu.se at $21{ }^{\circ} \mathrm{C}$, light schedule was $12 \mathrm{~h}$ daylight starting at 6.00 a.m. and $12 \mathrm{~h}$ darkness starting at 6.00 p.m., and the humidity was kept at $50 \%$. All testing was performed between 8.00 a.m. and 11.00 a.m. The rats were anesthetized with Isofluran ${ }^{\circledR}$ inhalation (isoflurane, Baxter Medical AB, Kista. Sweden). Before surgery, the rats received $0.04 \mathrm{ml}$ of Temgesic ${ }^{B}$ (buprenorphine $0.3 \mathrm{mg} / \mathrm{ml}$, Schering-Plough, Stockholm, Sweden) to reduce postoperative pain. The local animal ethics committee approved the experimental protocol.

\section{Dorsal Approach}

Disc scratch $(\mathrm{n}=10)$ : Following a $30 \mathrm{~mm}$ midline incision in the back, the left facet joint between the 4th and the 5th lumbar vertebra was removed and the 4th lumbar dorsal root ganglion and the 5th lumbar nerve root, including the intervertebral disc between the 4th and 5th lumbar vertebrae, were visualized. The surface of the disc was gently scraped by an injection needle to induce a superficial disc injury but with great care considering the leakage of nucleus pulposus should not occured. The spinal muscles were sutured and the skin closed by metal-clips.

Application of nucleus pulposus ( $\mathrm{n}=10)$ : Using the same exposure as in the sham series, which is about $10 \mathrm{mg}$ of nucleus pulposus, approximately the content of one rat lumbar disc, from a donor rat was placed on the L4-5 disc. The spinal muscles were sutured and the skin closed by metal-clips.

Historical controls: To relate the recorded behaviors following disc scratch and nucleus pulposus application data 
from a previous study was used [1]. In the first group 10 rats had a sham exposure of the disc between the 4th and 5th lumbar vertebrae ( $\mathrm{n}=10$, Control). In the other group the rats had the disc between the 4th and 5th lumbar vertebrae punctured by a $0.4 \mathrm{~mm}$ in diameter injection needle $(\mathrm{n}=10$, Disc puncture). Visual confirmation was obtained that there was a leakage of nucleus pulposus.

\section{Ventral Approach}

Control: The rat was placed on the back and the abdomen was opened through a midline incision. The disc between the 4 th and 5th lumbar vertebrae was carefully exposed from the left side of the aorta. No further procedures were performed in this group $(\mathrm{n}=10)$. The abdomen was closed by sutures and clips.

Disc puncture: The disc between the 4th and 5th lumbar vertebrae was exposed and punctured by a $0.4 \mathrm{~mm}$ diameter injection needle $(\mathrm{n}=10)$. Visual confirmation was obtained that there was a leakage of nucleus pulposus. The abdomen was closed by sutures and clips.

After surgery each specific rat received a unique identification number to allow for a blinded behavioral assessment.

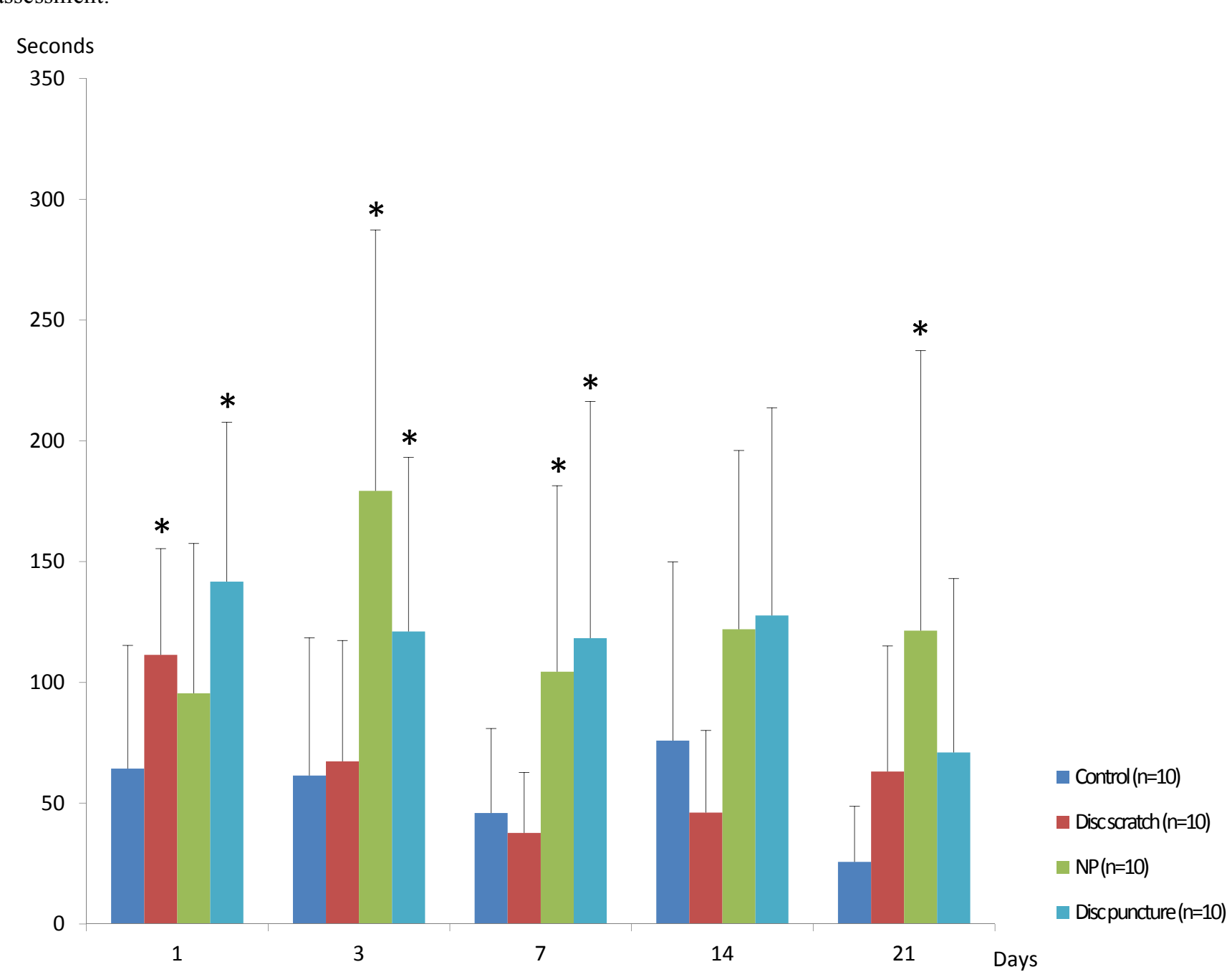

Fig (1). Bar graphs of grooming following the dorsal approach. Behaviors are expressed as mean \pm standard deviation. Asterisks indicate statistically significant changes between sham and a specific series. For comments see text.

\section{BEHAVIORAL TESTING}

Behavioral analyses were performed on days 1,3, 7, 14 and 21 after surgery. The rats were randomly placed in one of 4 boxes in a wooden framework placed on a transparent acrylic plate [9]. The rats were videotaped from below by a fixed video camera. The tape was started just before the rats were placed in their respective box. The rats were videotaped for slightly more than 10 minutes.

A blinded assessment was performed of the videorecordings. The duration of grooming was determined during a 10-minute observation time. "Wet-dog shakes" is a behavior that resembles a wet dog that is shaking to remove water from the fur. This behavior was recorded as number of episodes during the ten-minute observation time and was assessed in parallel to the recording of grooming.

The data were analyzed statistically regarding differences between the experimental groups and control at each day and for each behavior using ANOVA with Fishers PLSD at 5\%. The analyses were separated between the dorsal and the ventral approach groups. and 


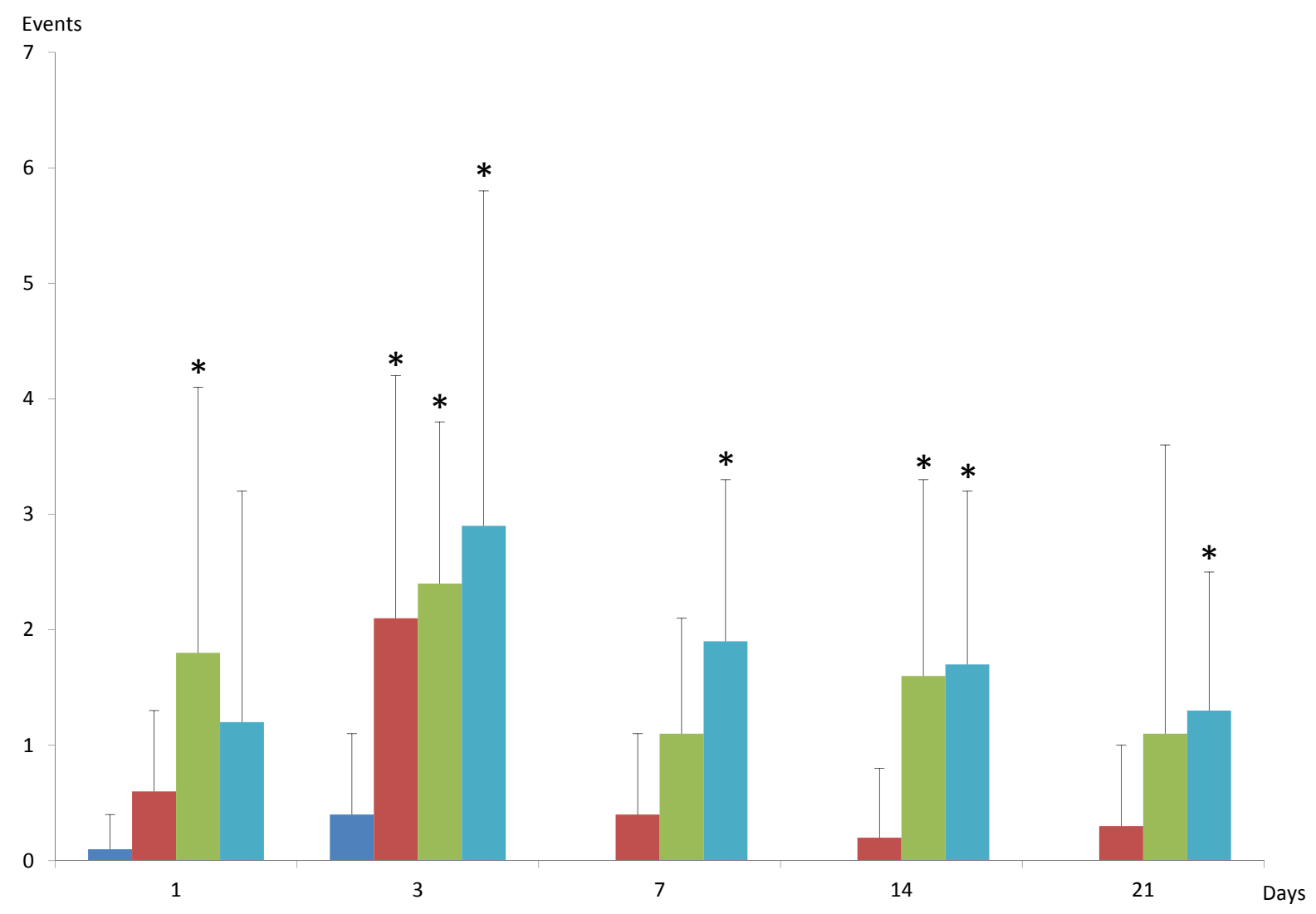

Fig. (2). Bar graph of "wet-dog shakes" following the dorsal approach (mean \pm SD). Asterisks indicate statistically significant changes between sham and a specific series. For comments see text.

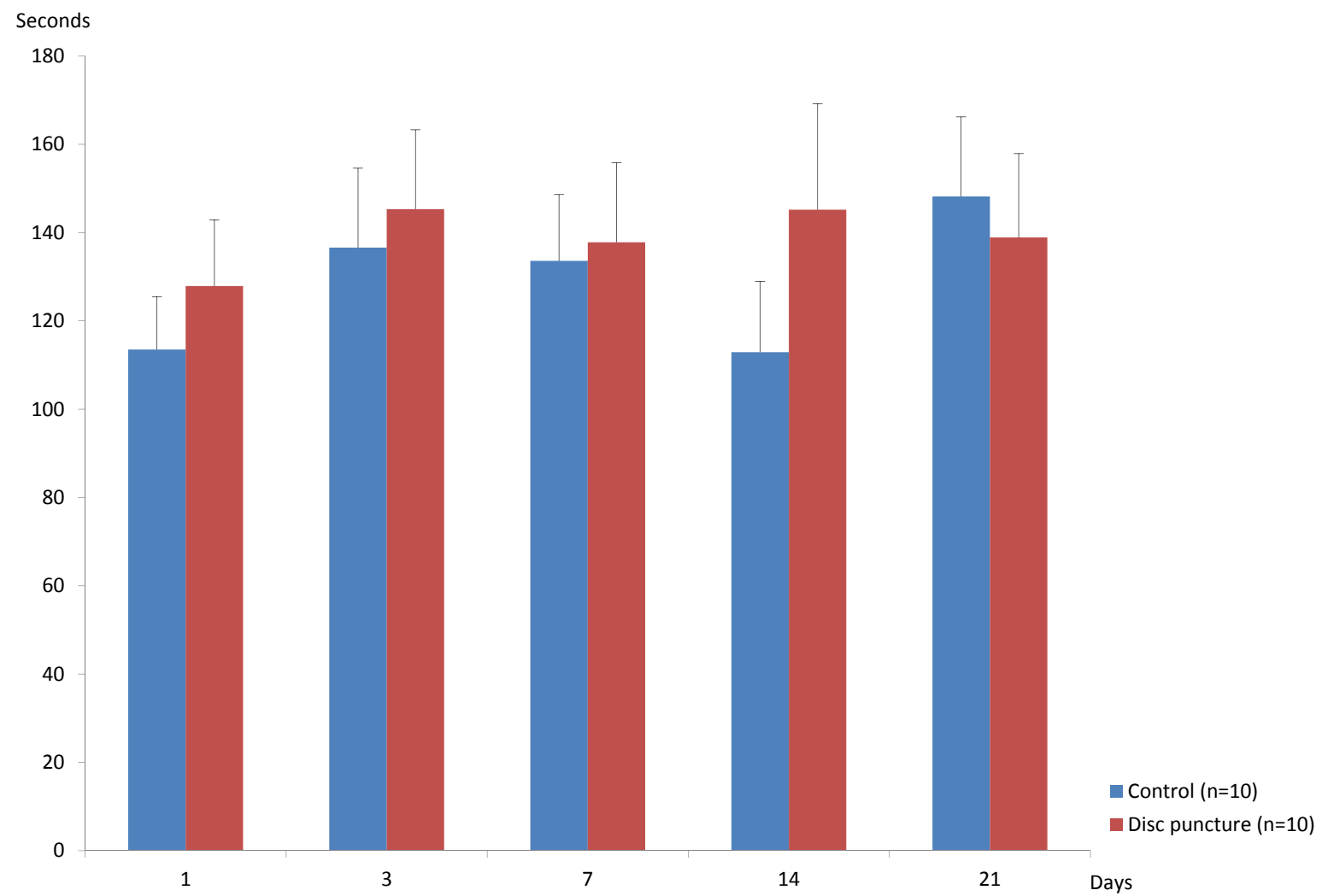

Fig. (3). Bar graphs of grooming following the ventral approach. Behaviors are expressed as mean \pm standard deviation. There are no statistically significant changes between sham disc puncture. 


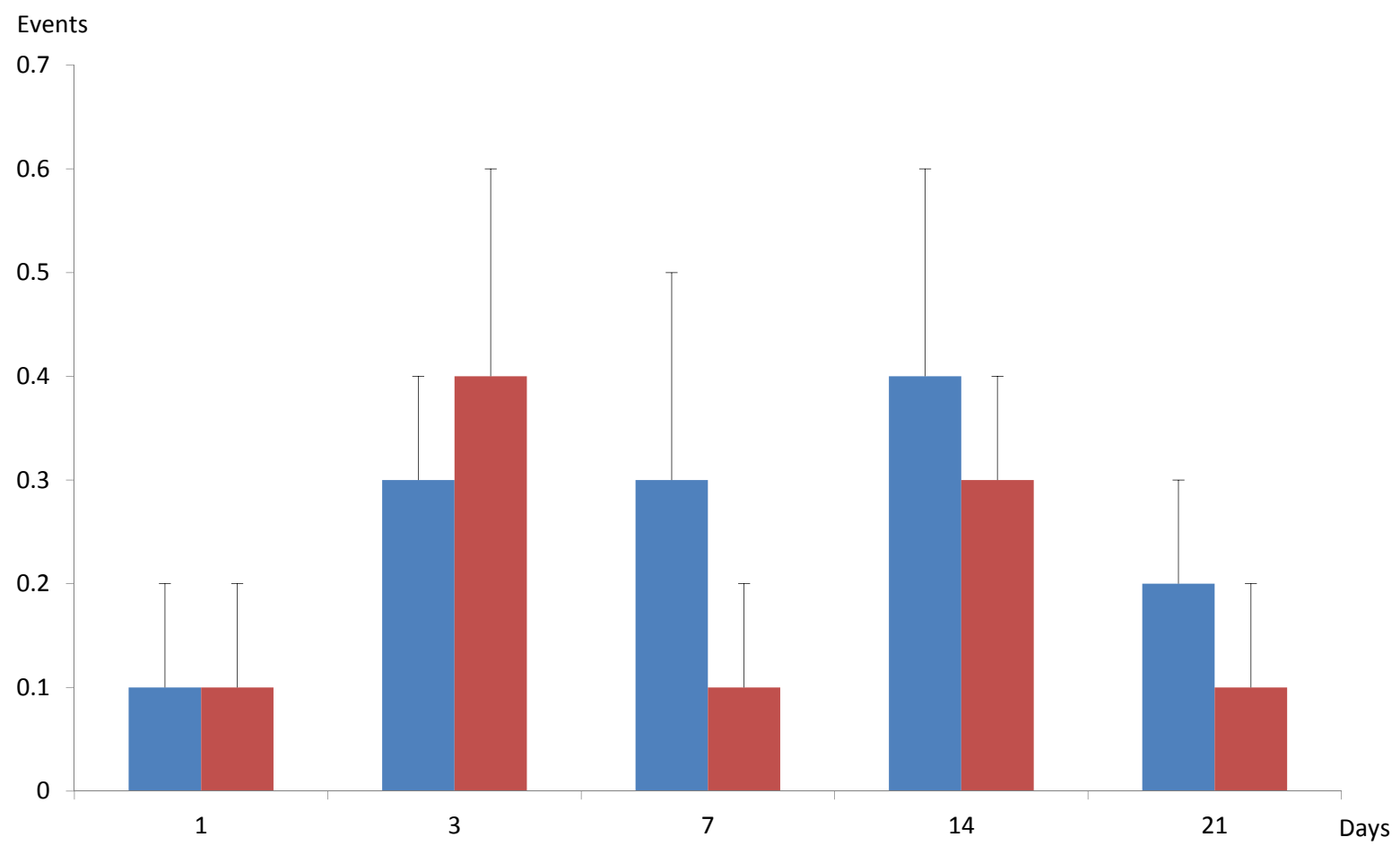

Fig. (4). Bar graph of "wet-dog shakes" following the ventral approach (mean $\pm \mathrm{SD}$ ). There are no statistically significant changes between sham disc puncture. (Please also note that the scale of the y-axis is 10-fold lower than in Fig. (2)).

\section{RESULTS}

All rats displayed good general condition throughout the 21 days of observation and no apparent signs of pain were noted by the naked eye.

\section{Dorsal Approach}

Grooming: At day 1 disc scratch and disc puncture showed statistically significantly increased grooming compared to control (Fig. 1). Application of nucleus pulposus demonstrated an increased grooming duration but it was not statistically significant. At day 3, application of nucleus pulposus showed a pronounced increase in grooming and was statistically significantly different to control together with disc puncture. Disc scratch showed comparable grooming to control. The same pattern was found at day 7 although application of nucleus pulposus as well as disc puncture had similar grooming duration. The data at day 14 was similar to day 7 but the changes recorded were not statistically significant. At day 21 post-surgery, "disc scratch" induced increased grooming comparable to disc puncture. Application of nucleus pulposus, however, showed a statistically significant increase in grooming that was more pronounced than for disc scratch and disc puncture.

Wet dog shakes: At day 1, application of nucleus pulposus induced the highest incidence of WDS and was also statistically significant to control (Fig. 2). Both disc scratch and disc puncture induced an increased incidence in WDS but without any statistical significance. The third day, all three groups induced statistically significantly higher incidence than control. At days 7 to 21 , disc scratch was no longer statistically significantly different to control. Application of nucleus pulposus induced a slight increase at day 7 and increases similar to disc puncture at day 14 and 21 , however, only statistically significant at day 14 . Disc puncture induced statistically significant number of increase in WDS counts at days 7, 14 and 21 .

\section{Ventral Approach}

Grooming: In the ventral approach series, the duration of grooming was similar between sham and disc puncture except for day 14 where disc puncture induced a slightly higher duration than control (Fig. 3). There were no statistically significant differences noted between control and disc puncture at any of the studied days.

Wet dog shakes: The incidence of WDS increased during days 3, 7 and 14 in both groups except for disc puncture at day 7 which showed baseline level. At day 21 the incidence was almost back to baseline. At days 7, 14 and 21 the incidence of WDS was higher for control than for disc puncture. There were no statistically significant differences noted between control and disc puncture at any of the studied days (Fig. 4).

\section{DISCUSSION}

Behavior analysis demonstrated that disc puncture and application of nucleus pulposus resulting in nucleus pulposus exposure to the spinal canal and adjacent nerve root is more likely to induce changes in grooming and wet dog shakes (WDS) then a dorsal disc scratch is. Puncture of the ventral aspect of the disc did not induce any behavioral changes compared to sham.

A recent experimental study assessed that if there is a controlled puncture of a lumbar disc in the rat, then 
mimicking an annular tear, might produce behavioral changes, studied by spontaneous pain behavior recordings [1]. The data showed that there was an increase in grooming and wet dog shakes in the disc-punctured rats. It is known that an increase in grooming has been suggested to reflect anxiety, stress and chronic neuropathic pain [2-8]. Assessment of wet dog shakes (WDS) has been widely used in psychopharmacological studies and is a typical sign of withdrawal from opiates, benzodiazepines and barbiturates [10-14]. WDS has also been considered to indicate stress $[15-17]$ and pain $[18,19]$ and is regulated by 5 -Ht-activation [20-22]. Although it was not possible to draw any conclusions if grooming and WDS could correspond to disc induced low back pain, it was concluded that the findings seemed to indirectly support such hypothesis [1].

From the previous study it was not clarified if the observed changes were induced by the disc injury or by the presence of nucleus pulposus [1]. To assess this question the present study was undertaken where additional series had either only a superficial disc injury with no penetration to the nucleus pulposus or nucleus pulposus from a donor rat placed on the disc surface without simultaneous disc injury. Based on the knowledge that the ventral aspect of the disc is poorly innervated by sensory fibers, a group with ventral disc puncture was included with its own control group [2326]. The assumption was that due to the poor innervation, ventral disc puncture would not induce any behavioral changes. Due to the major undertakings of the video analyses it was decided to use two groups from a previous study [1] for comparison. This might be a limitation of the present study but since the general procedures were identical between previous and present experimental series it was considered to be justified.

The data for the ventral approach showed that there was no or only minor differences between sham and disc puncture. Assuming that sham exposure would not induce behavior changes, puncture of the ventral aspect of the disc thus did not seem to induce any behavioral changes. This may also indicate that the disc injury obtained at disc puncture per se does not induce behavioral changes. Since the innervation of the ventral aspect of the disc is known to be sparse and predominantly sympathetic compared to the dorsal aspect [23-26], one may suppose that behavior changes observed previously following puncture of the dorsal aspect [1] may relate to the innervation type and density.

The general appearance of behavior changes following the dorsal procedures seemed to indicate that the presence of nucleus pulposus in the epidural space is more likely to induce changes than the disc injury per se. In both the groups the disc injury and the disc puncture (historical), group there was a disc injury. In the disc scratch group the injury was only superficial and did not allow for leakage of nucleus pulposus. Anyway, disc puncture induced more pronounced changes than the disc scratch group. This might of course relates not only to the degree of disc injury but also to the presence or absence of free nucleus pulposus material in the epidural space. In the group with applied nucleus pulposus nucleus pulposus was present in the epidural space but there was no disc injury. Since the changes induced by nucleus pulposus application was similar to the changes induced by disc puncture it seems more likely that the changes would relate to epidurally present nucleus pulposus material then the actual injury to the disc.

Previous studies have suggested that bioactive components of the nucleus pulposus, in particular TNF, may induce behavioral changes in the present model $[1,9]$. A recent study using the present disc puncture model also clearly demonstrated that treatment with both non-specific TNF inhibitor (doxycycline) and a specific TNF-inhibitor (infliximab) reduced the incidence of WDS [27]. This study may further support the impression that the behavioral changes induced by disc puncture relates to the activity of TNF and thus to the presence of epidural nucleus pulposus rather than to the disc puncture per se.

A further possible limitation of the present study is that no pre-surgical behavior assessments were performed. In the past, it has been verified that sham surgery from the dorsal aspect does not induce any changes and comparisons have been performed day by day. In the present study, a ventral approach was also introduced. It was noted that the duration of grooming in general was higher in the ventral sham group compared to the dorsal sham group, while incidence of WDS was comparable. In retrospect, it would have been interesting to see if there was an increase in grooming duration from a pre-operative assessment to day 1 in the ventral sham group. However, since the grooming duration was similar between sham and disc puncture in the ventral series at day 1 it seems likely that it would have been an effect of surgery rather than obscuring any behavioral changes induced by the ventral disc puncture.

In conclusion, the data from the study indicate that behavioral changes induced by disc puncture on the posterior aspect of the disc are more likely to relate to the epidural presence of nucleus pulposus than the disc injury per se.

\section{ACKNOWLEDGEMENTS}

This work was supported by grants from the Swedish Research Council (521-2007-2956), the Gothenburg Medical Society, AFA Insurance, the Gothenburg Medical Society, Ingabritt and Arne Lundberg Research foundation, the Ollie and Elof Ericsson Foundation for Scientific Research, Stiftelsen Olle Engkvist byggmästare and the Felix Neubergh Foundation.

\section{CONFLICT OF INTEREST}

The corresponding author is partly owner and CEO of the companies of Orthopaedic R\&D AB and Sciaticon AB that both hold patents for treatment of spinal pain conditions by TNF-inhibition.

\section{REFERENCES}

[1] Olmarker K. Puncture of a lumbar intervertebral disc induces changes in spontaneous pain behavior: an experimental study in rats. Spine 2008; 33(8): 850-5.

[2] Millan MJ, Emrich HM. Endorphinergic systems and the response to stress. Psychother Psychosom 1981; 36(1): 43-56.

[3] Crawley JN, Moody TW. Anxiolytics block excessive grooming behavior induced by ACTH1-24 and bombesin. Brain Res Bull 1983; 10(3): 399-401.

[4] De Castro-Costa M, Gybels J, Kupers R, Van Hees J. Scratching behaviour in arthritic rats: a sign of chronic pain or itch? Pain 1987; 29(1): 123-31. 
[5] Vos BP, Strassman AM, Maciewicz RJ. Behavioral evidence of trigeminal neuropathic pain following chronic constriction injury to the rat's infraorbital nerve. J Neurosci 1994; 14(5 Pt 1): 2708-23.

[6] Abbott FV, Franklin KB, Westbrook RF. The formalin test: scoring properties of the first and second phases of the pain response in rats. Pain 1995; 60(1): 91-102.

[7] Deseure KR, Adriaensen HF. Comparison between two types of behavioral variables of non-evoked facial pain after chronic constriction injury to the rat infraorbital nerve. Comp Med 2002; 52(1): 44-9.

[8] Eriksson J, Jablonski A, Persson AK, et al. Behavioral changes and trigeminal ganglion sodium channel regulation in an orofacial neuropathic pain model. Pain 2005; 119(1-3): 82-94.

[9] Olmarker K, Storkson R, Berge OG. Pathogenesis of sciatic pain: a study of spontaneous behavior in rats exposed to experimental disc herniation. Spine 2002; 27(12): 1312-7.

[10] Colasanti B, Khazan N. Imipramine-induced changes in the rapid eye movement sleep rebound and wet dog shakes of morphineabstinent rats. Neuropharmacology 1975; 14(5-6): 361-7.

[11] Nakamura H, Ishii K, Shimizu M. Some altered responses in rats formerly dependent on morphine. Psychopharmacology (Berl) 1978; 56(3): 269-77.

[12] Baldino F, Jr., Cowan A, Geller EB, Adler MW. Effects of antipsychotic and antianxiety drugs on the morphine abstinence syndrome in rats. J Pharmacol Exp Ther 1979; 208(1): 63-6.

[13] Horowitz GP, Allan AM. Morphine withdrawal in mice selectively bred for differential sensitivity to ethanol. Pharmacol Biochem Behav 1982; 16(1): 35-9.

[14] Martin WR, McNicholas LF, Cherian S. Diazepam and pentobarbital dependence in the rat. Life Sci 1982; 31(8): 721-30.

[15] Treptow K, Bondarenko NA, Waldmann AW, Oehme P. [The effect of substance $\mathrm{P}$ on the swimming behavior of rats under stressing and nonstressing conditions]. Pharmazie 1986; 41(8): $578-80$.

[16] Deschamps K, Couture R. The ventral tegmental area as a putative target for tachykinins in cardiovascular regulation. Br J Pharmacol 2005; 145(6): 712-27.

[17] Brotto LA, Hanson LA, Gorzalka BB. Nefazodone attenuates the stress-induced facilitation of wet dog shaking behaviour but not the facilitation of sexual behaviour in female rats. Eur J Pharmacol 1999; 381(2-3): 101-4.

[18] Papir-Kricheli D, Frey J, Laufer R, et al. Behavioural effects of receptor-specific substance $P$ agonists. Pain 1987; 31(2): 263-76.

[19] Kitamura Y, Kitagawa K, Fujitani Y, et al. The 5-HT(1A) receptor full agonist, 8-OH-DPAT inhibits ACTH-induced 5-HT(2A) receptor hyperfunction in rats: involvement of 5-HT(1A) receptors in the DOI-induced wet-dog shakes in ACTH-treated rats. Biol Pharm Bull 2007; 30(1): 117-20.

[20] Fozard JR, Palfreyman MG. Metoclopramide antagonism of 5hydroxytryptophan-induced \& quot; wet-dog\&quot; shake behaviour in the rat. Naunyn Schmiedebergs Arch Pharmacol 1979; 307(2): 135 42.

[21] Olivier B, Mos J, van Oorschot R, Hen R. Serotonin receptors and animal models of aggressive behavior. Pharmacopsychiatry 1995; 28 Suppl 2: 80-90.

[22] Otoom SA, Handu SS, Wazir JF, et al. Veratridine-induced wet dog shake behaviour and apoptosis in rat hippocampus. Basic Clin Pharmacol Toxicol 2006; 98(4): 423-6.

[23] Bogduk N, Tynan W, Wilson AS. The nerve supply to the human lumbar intervertebral discs. J Anat 1981; 132(Pt 1): 39-56.

[24] Tsuji H, Hirano N, Ohshima H, Ishihara H, Terahata N, Motoe T. Structural variation of the anterior and posterior anulus fibrosus in the development of human lumbar intervertebral disc. A risk factor for intervertebral disc rupture. Spine (Phila Pa 1976) 1993; 18(2): 204-10.

[25] Wong-Chung JK, Naseeb SA, Kaneker SG, Aradi AJ. Anterior disc protrusion as a cause for abdominal symptoms in childhood discitis. A case report. Spine (Phila Pa 1976) 1999; 24(9): 918-20.

[26] Takahashi Y, Aoki Y, Douya H, Ohtori S, Takahashi K. Projection field of primary afferent fibers innervating the ventral portion of the lumbar intervertebral disc in the spinal cord dorsal horn. Anat Sci Int 2006; 81(2): 92-9.

[27] Nakamae T, Ochi M, Olmarker K. Pharmacological inhibition of tumor necrosis factor may reduce pain behavior changes induced by experimental disc puncture in the rat: an experimental study in rats. Spine (Phila Pa 1976) 2011; 36(4): E232-6.

(C) Nilsson et al.; Licensee Bentham Open.

This is an open access article licensed under the terms of the Creative Commons Attribution Non-Commercial License (http://creativecommons.org/licenses/by$\mathrm{nc} / 3.0 /$ ) which permits unrestricted, non-commercial use, distribution and reproduction in any medium, provided the work is properly cited. 